
cultural y científica entre Perú y Francia

\title{
Un taller y un seminario consagrados a la investigación sobre los riesgos y su aplicación (Quito, noviembre de 2006)
}

Robert D’Ercole, Pascale Metzger, Nury Bermúdez y Sebastián Hardy

\section{(2) OpenEdition}

Edición electrónica

URL: http://journals.openedition.org/bifea/4701

DOI: 10.4000/bifea.4701

ISSN: 2076-5827

Editor

Institut Français d'Études Andines

Edición impresa

Fecha de publicación: 8 mayo 2007

Paginación: 171-173

ISSN: 0303-7495

Referencia electrónica

Robert D’Ercole, Pascale Metzger, Nury Bermúdez y Sebastián Hardy, « Un taller y un seminario consagrados a la investigación sobre los riesgos y su aplicación (Quito, noviembre de 2006) », Bulletin de l'Institut français d'études andines [En línea], 36 (1) | 2007, Publicado el 08 mayo 2007, consultado el 02 diciembre 2020. URL : http://journals.openedition.org/bifea/4701 ; DOI : https://doi.org/10.4000/ bifea.4701

(lis Bulletin de l'Institut français d'études andines sont mis à disposition selon les termes de la licence Creative Commons Attribution - Pas d'Utilisation Commerciale - Pas de Modification 4.0 International. 


\section{Eventos}

UN TALLER Y UN SEMINARIO CONSAGRADOS A LA INVESTIGACIÓN SOBRE LOS RIESGOS Y SU APLICACIÓN (QUITO, NOVIEMBRE DE 2006)

\section{PROBLEMÁTICA Y OBJETIVOS}

A escala mundial, durante estas últimas décadas ha aumentado fuertemente el conocimiento de los procesos físicos y humanos que originan catástrofes; paralelamente, hemos asistido al desarrollo de los medios técnicos que permiten preverlas e intervenir de manera preventiva. Pero al mismo tiempo, se observa un aumento muy sensible de las víctimas y de las pérdidas materiales y financieras cuando se producen estas catástrofes. Por otro lado, si la producción de conocimientos en materia de riesgo puede ser considerada como abundante, esta desemboca en conclusiones escasamente tomadas en cuenta por los decididores en el marco de políticas de manejo de riesgos. Se puede entonces considerar que la investigación no permite reducir las vulnerabilidades y los riesgos de manera eficaz. Esta paradoja y estas distorsiones revelan un cierto malestar en la relación existente entre la investigación científica y su aplicación en materia de reducción de riesgos. Para reflexionar en torno a esta relación, junto con las contrapartes operacionales, en noviembre de 2006 se organizaron en Quito dos eventos complementarios.

\section{Taller del 14 de noviembre de 2006 (Centro Cultural Metropolitano, Quito) El programa IRD/MDMQ «Sistema de Información y Riesgos en el Distrito Metropolitano de Quito». ¿Qué tipo de investigación? ¿Para qué tipo de usos?}

Este taller tenía por objetivo reflexionar acerca de la relación investigación/aplicación a partir de un ejemplo concreto: una investigación realizada entre 1999 y 2004 por el IRD en colaboración con la Municipalidad de Quito dentro del marco del programa «Sistema de Información y Riesgos en el distrito Metropolitano de Quito (DMQ)». Esta investigación ha permitido desarrollar una metodología original de análisis de la vulnerabilidad del distrito de Quito y proponer medidas preventivas útiles para los planificadores urbanos y gestionarios de riesgos.

El taller fue organizado y financiado por el IRD, la Municipalidad de Quito, el IFEA y el equipo de investigadores ecuatorianos PAUD (Por el Ambiente Urbano y Desarrollo). Reunió a cincuenta personas provenientes de la Municipalidad y de instituciones públicas o privadas implicadas en la investigación o usuarias. El primer medio día fue consagrado a la investigación y su aplicación en el campo de la prevención y la planificación preventiva urbana en el DMQ. En el segundo medio día se trató sobre la investigación y su aplicación en el manejo de las crisis en el DMQ. En ambos casos, una mesa redonda y un debate público siguieron a la presentación de las principales conclusiones de la investigación efectuada por el IRD y la Municipalidad de Quito. 


\title{
Seminario-taller de los días 16 y 17 de noviembre de 2006 (Hotel Dann Carlton, Quito)
}

\author{
Vulnerabilidad y riesgos de origen natural y antrópico. ¿Qué tipo de investigación? \\ ¿Para qué tipo de usos?
}

En relación al taller del 14 de noviembre, este segundo encuentro apuntaba a ampliar el debate sobre la relación investigación/aplicación en el campo de los riesgos. Los 98 participantes representaban una gama muy variada de actores del conocimiento y del manejo de riesgos y de crisis, entre los cuales científicos, técnicos y responsables de la gestión territorial (políticos y administrativos), financistas, organismos internacionales, ONG, medios de comunicación, etc.

Se trataba de reflexionar sobre la relación investigación/aplicación a partir de experiencias concretas de estudios de investigación desarrollados durante los últimos años en Ecuador para llegar a proposiciones concretas que permitan realizar una investigación más útil, más aplicable en materia de riesgos.

Este seminario fue organizado y financiado por el IRD, la Municipalidad de Quito, el IFEA, el equipo de investigadores ecuatorianos PAUD (Por el Ambiente Urbano y Desarrollo) y la ONG italiana COOPI (Cooperazione Internazionale). Los conferencistas intervinieron durante las mañanas para exponer su propia experiencia. Por las tardes, los talleres fueron organizados en torno a ocho grupos de trabajo para favorecer los intercambios directos, la toma de la palabra, y dar cuenta de la diversidad de las experiencias. En la primera jornada, los grupos de trabajo se centraron en los problemas encontrados en materia de aplicación de la investigación sobre la vulnerabilidad y los riesgos. El objetivo de la segunda jornada fue de extraer proposiciones que permitan mejorar la aplicabilidad de esta investigación. Cada grupo de trabajo pudo expresar su punto de vista restituyendo los resultados de sus reflexiones al conjunto de los participantes.

\section{ALGUNAS CONCLUSIONES PRELIMINARES DE ESTOS DOS ENCUENTROS}

Las reflexiones efectuadas durante los dos eventos serán analizadas con precisión y serán objeto de publicaciones. Sin embargo, se pueden sacar algunas conclusiones preliminares.

Ambos eventos han sido exitosos por el número y la diversidad de los participantes, el interés de estos últimos por la temática propuesta y la calidad de su participación. Han constituido espacios de intercambio y de discusión no solo entre investigadores y técnicos sino también entre los diversos actores de la planificación, de la prevención y del manejo de las crisis, los mismos que tienen raras ocasiones de encontrarse y de reflexionar juntos.

Entre los problemas evocados que explican los límites de la aplicación de la investigación figuran la comunicación y la difusión de esta investigación. Las lagunas han sido puestas en evidencia en materia de cartografía del riesgo o de sus diferentes componentes: esta se dirige habitualmente a un público especializado muy a menudo excluyente de los actores decisivos para la acción, en particular los políticos y la población.

Pero los problemas no se limitan a los conocimientos producidos y a la comunicación. En efecto, las diferentes experiencias presentadas y las reflexiones que las han acompañado han mostrado nítidamente que una investigación sobre los riesgos que se quiere útil y aplicable debe ser concebida dentro de un marco participativo que implique no solamente a los diversos actores de la gestión territorial y de riesgos, sino también a las comunidades expuestas a estos riesgos y «destinatarias» de las políticas de prevención. En efecto, las poblaciones constituyen frecuentemente objetos de estudio pero en general no están implicadas en la investigación. Por otro lado, los gestionarios aparecen antes que nada como los «clientes» de los científicos y no como verdaderas contrapartes. Este enfoque participativo que permite unir el conocimiento 
científico y las prácticas de gestión supone una reconsideración de la manera según la cual se construye, se valida y se realiza una investigación, en particular en los países en desarrollo.

La reflexión efectuada sobre la utilidad de investigaciones recientes ha mostrado que no solamente son útiles las informaciones factuales (datos, mapas, etc.). Las investigaciones efectuadas en un marco participativo, como aquella referente a la vulnerabilidad del Distrito Metropolitano de Quito, permiten a las contrapartes no científicas movilizar y apropiarse conceptos, enfoques y métodos que dan más sentido a su acción y la hacen más eficaz. Este ha sido, en particular, el caso con las direcciones metropolitanas de planificación territorial y de transporte del MDQ con las cuales los investigadores trabajaron de manera estrecha y duradera. En retorno, la experiencia, las prácticas y las necesidades de las contrapartes permiten no solo alimentar la investigación sino también formularla de otra manera, incluso de retroceder hasta los conceptos.

Robert D'ERCOLE, Pascale METZGER, Nury BERMÚDEZ, Sebastián HARDY 